\title{
嫦娥五号任务中的多通道多比特VLBI信号采集 记录与传输一体化技术
}

朱人杰 ${ }^{1,2,3,4}$, 吴亚军 ${ }^{1,2}$, 郑为民 ${ }^{1,2,3,4^{*}}$, 李纪云 ${ }^{1}$, 郭绍光 ${ }^{1,2}$, 张秀忠 ${ }^{1}$, 王广利 ${ }^{1,4}$

1. 中国科学院上海天文台, 上海 200030 ;

2. 中国科学院上海天文台, 中国科学院射电天文重点实验室, 南京 210023;

3. 国家基础学科公共科学数据中心, 北京 100190 ;

4. 中国科学院上海天文台, 上海市空间导航与定位技术重点实验室, 上海 200030

*联系人, E-mail: zhwm@shao.ac.cn

收稿日期: 2021-03-19; 接受日期: 2021-05-27; 网络出版日期: 2021-08-05

国家重点研发计划(编号: 2018YFA0404704)和探月工程项目资助

摘要 对接收到的观测信号进行实时采集记录与传输是甚长基线干涉测量(VLBI)的重要技术环节。在嫦娥五号 任务中, VLBI终端需要同时采集记录轨道器、上升器、着陆器等多个目标探测器发出的测控信号、数传信号并 分时采集记录河外射电源信号. 由于探测器信号频点多、动态范围大、频谱形式复杂, 现有天文VLBI终端不能 满足探月与深空探测的需求. 我们针对嫦娥五号探测器信标特点, 开展了多通道、多比特信号采集、记录与传输 一体化技术研究; 提出了可变频率多项滤波器组算法, 可在很宽的频段范围内, 灵活设置信号频点, 并能获得平坦 的幅度特性与线性相位特性. 采用该技术研制的新型采集记录与传输一体化VLBI终端, 具备16 个基带通道, 能够 实时采样、记录嫦娥五号多个探测器用于VLBI测轨的全部测控与数传信标, 并能够通过网络将数据实时传送至 VLBI中心, 确保了在嫦娥五号多种信标复杂电磁环境下的VLBI测轨。相比以往天文终端, 其实时性、测量精度和 可靠性都有大幅提高, 极大地支持了我国嫦娥五号首次月球采样返回关键段测轨任务的圆满完成. 为适应未来射 电源与探测器的宽窄带观测需求, 新型采集记录与传输一体化VLBI终端还具备多比特量化功能.

关键词 VLBI, 嫦娥五号, 可变频率多项滤波器组算法, 时延, 一体化终端

PACS: 95.55.Br, 95.55.Jz, 95.55.Pe, 95.75.Wx

\section{1 引言}

甚长基线干涉测量(Very Long Baseline Interferometry, VLBI) 是1967年诞生的一种新的射电干涉技 术 $^{[1]}$. 它采用独立本振和干涉方法, 将多个距离数百乃
至数千公里的射电望远镜，合成为巨大的综合孔径望 远镜，其最大等效直径等于望远镜之间的最长距离, 是目前分辨率最高的天文观测技术. VLBI能获得优于 亚毫角秒量级的测角分辨率, 相当于在地球上能分辨 出月球上篮球大小的面积, 也能够以毫米的精度测量

引用格式: 朱人杰, 吴亚军, 郑为民, 等. 嫦娥五号任务中的多通道多比特VLBI信号采集记录与传输一体化技术. 中国科学: 物理学 力学 天文学, 2021, 51: 119506

Zhu R J, Wu Y J, Zheng W M, et al. Integration of very long baseline interferometry data acquisition, recording, and transmission with multichannel and multibit for CE-5 mission (in Chinese). Sci Sin-Phys Mech Astron, 2021, 51: 119506, doi: 10.1360/SSPMA-2021-0076 
上千公里的基线长度 ${ }^{[2]}$.

以VLBI终端为代表的VLBI信号采集、记录和传 输技术，是VLBI核心技术之一. 它将射电望远镜射频 接收机输出的中频信号转换到基带，以二进制的形式 记录在介质上，并送到数据处理中心. VLBI测量精度 的提高，离不开VLBI信号采集、记录和传输技术的 进步 ${ }^{[3]}$.

国际射电天文界出于天体物理和天体测量的观测 需求, 一直在不断发展VLBI信号采集、记录和传输技 术. 目前VLBI终端已经历了从模拟到数字，从窄带到 宽带的数代发展.

近十年来，各个国家或地区都相继研制了多种数 字终端, 如美国的RDBE (ROACH Based Digital Back End) ${ }^{[4]}$ 系列、欧洲的DBBC (Digital Base Band Convertor) $^{[5]}$ 系列、日本的ADS (Advanced Data Sampler) ${ }^{[6]}$ 系列. 上海天文台也为中国VLBI网研制了CDAS (Chinese VLBI Data Acquisition System $)^{[7]}$ 系列终端, 国内 装备学院也为深空网研制过干涉测量终端 ${ }^{[8]}$.

天文观测的目标为遥远的河外射电源, 其信号为 宽带白噪声, 极其微弱, 为获得较高的信噪比, 在天线 口径已经确定的情况下, 需要增加带宽. 为满足天文观 测日益增长的灵敏度需求, 用于天文的VLBI终端一直 追求更大的带宽, 目前已可达到4-8 GHz带宽, 数据率 可达8-16 Gbps. 毫米波VLBI阵列EHT的数据率甚至 达到了 $64 \mathrm{Gbps}^{[9]}$.

对于射电源信号, 在满足总数据率约束条件下, 为 尽可能增加带宽, 在数据采集时, 一般采用 1 bit或 2 bit 量化. 相对而言, 探测器信标发送带宽有限信号. 比如, 嫦娥五号等探测器用于VLBI观测的DOR (Differential One-way Ranging)信标, 就包含了两组点频信号 ${ }^{[10]}$.
DOR信标发出的每个点频信号带宽极窄，功率谱密度 很强. 若采用天文宽带观测模式, 则带宽使用效率必 然很低. 对于这种窄带单音信号, 若仍采用 1 bit或2 bit 量化, 则必然会带来较大的量化损失, 增大观测噪声.

针对深空探测信号的特点，美国国家航空航天局 和欧洲航天局分别设计了WVSR (Wide Band VLBI Science Receiver) ${ }^{[11]}$ 终端和IFMS (Intermediate Frequency And Modem System) ${ }^{[12]}$ 终端, 用于VLBI测轨.

表1比较了几类数字终端的功能特点, 可以看到, 传统的天文VLBI数字终端，基带信号最多以 2 bit输 出, 最小带宽在 $500 \mathrm{kHz}$, 而WVSR和IFSM分别采用了 8 bit和12 bit输出, 最小带宽可以到 $1 \mathrm{kHz}$.

在我国探月工程中，从CE-1至CE3以及CE-5T1任 务，中国VLBI网一直使用天文终端，并不能完全适应 CE-5和深空探测任务的需求, 存在着各种不足.

在嫦娥一号任务时, 各VLBI观测站沿用了国外的 模拟VLBI终端, 采用的是模拟变频和基带采样技术. 中频信号依次经过模拟变频器(图1(a))、采样器、格 式器和进口的Mark5 $\mathrm{A}^{[13]}$ 记录设备, 最终通过网络传输 到远程VLBI中心. 设备模块多, 进口设备一旦发生故 障, 维护不便.

从嫦娥二号任务开始，我们使用了自主研制的 CDAS数字终端和进口的Mark $5 \mathrm{~B}^{[14]}$ 记录设备, 如图 1(b)所示. 由于CDAS采用数字变频和中频采样技术, 替代了原先的模拟终端，但仍然存在单机模块多、可 靠性不高、使用不便等问题, 性能有待进一步提高.

此外, 由于CDAS数字终端的结构和算法的限制, 仪器各通道间的设备时延差异较大, 一致性较差, 对 测量精度有影响 ${ }^{[15]}$, 且不支持多比特量化模式. 而且 因为沿用了进口的Mark5B终端, 无法同时进行数据实

表 1 各类数字终端的比较

Table 1 Comparison of various digital backends

\begin{tabular}{|c|c|c|c|c|c|c|c|c|c|}
\hline 数字终端 & $\begin{array}{c}\text { 国家 } \\
\text { (地区) }\end{array}$ & 中频输入 & $\begin{array}{l}\mathrm{ADC} \text { 量 } \\
\text { 化位数 }\end{array}$ & $\begin{array}{c}\text { 采样频率 } \\
(\mathrm{MHz})\end{array}$ & $\begin{array}{c}\text { 基带通 } \\
\text { 道数 }\end{array}$ & $\begin{array}{l}\text { 基带输出量 } \\
\text { 化比特数 }\end{array}$ & 基带带宽 (MHz) & 数据格式 & 应用领域 \\
\hline RDBE2 & 美国 & $512 \mathrm{MHz}$ 带宽×2路 & 8 & 1024 & 16 & 2 & 32 & Mark5B/VDIF & 天文 \\
\hline $\mathrm{DBBC} 2$ & 欧洲 & $512 \mathrm{MHz}$ 带宽×4路 & 8 & 1024 & 16 & 2 & $0.5 / 1 / 2 / 4 / 8 / 16 / 32$ & Mark5B/VDIF & 天文 \\
\hline ADS3000+ & 日本 & $512 \mathrm{MHz}$ 带宽 ×4 路 & 8 & 1024 & 16 & 2 & $0.5 / 1 / 2 / 4 / 8 / 16 / 32$ & Mark5B & 天文 \\
\hline CDAS & 中国 & $512 \mathrm{MHz}$ 带宽×4路 & 8 & 1024 & 16 & 2 & $0.5 / 1 / 2 / 4 / 8 / 16 / 32$ & Mark5B & 天文、深空探测 \\
\hline WVSR & 美国 & 10-630 MHz×4路 & 8 & 1280 & 16 & 8 & $0.001-16$ & RDEF & 深空探测 \\
\hline IFMS & 欧洲 & 70 MHz带宽×8路 & 8 & 280 & 8 & 12 & $0.001-2$ & RDEF & 深空探测 \\
\hline
\end{tabular}


(a)

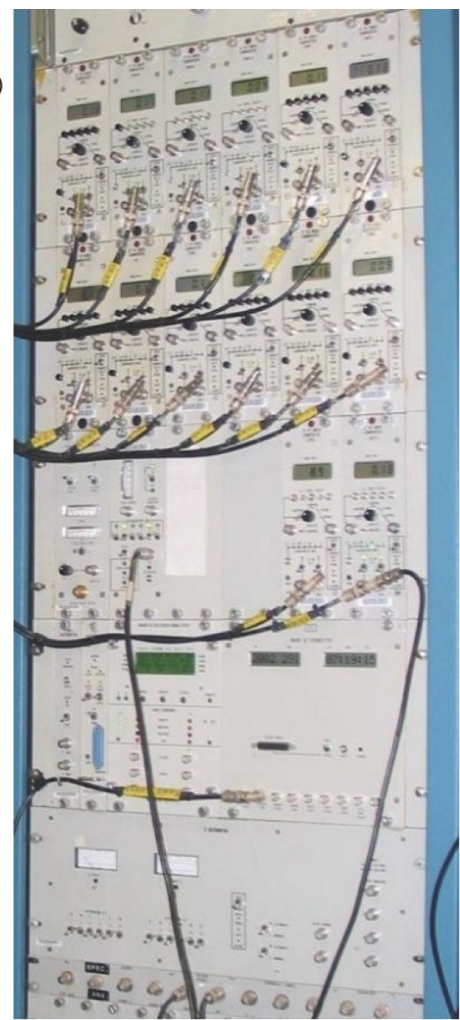

(b)
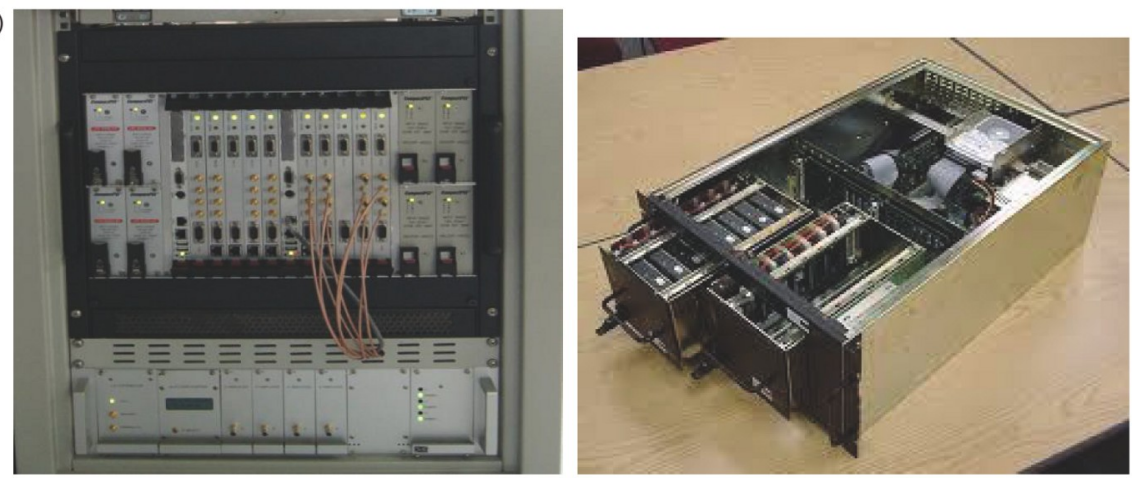

(c)

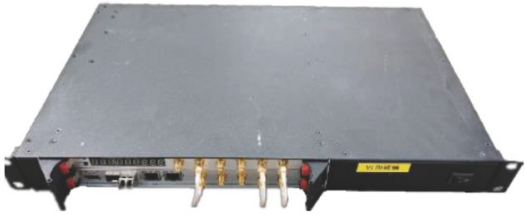

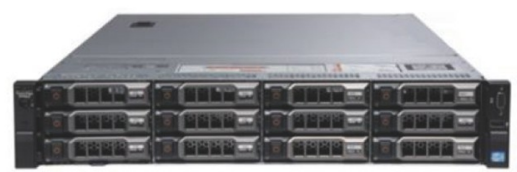

图 1 (网络版彩图)探月任务中VLBI信号采集技术的发展. (a) 模拟变频+基带采样技术; (b) 数字变频+中频采样技术; (c) 采 集、记录、传输一体化技术

Figure 1 (Color online) The development of VLBI signal acquisition technology in lunar mission. (a) Analog mixer + baseband sampler; (b) digital mixer + IF sampler; (c) integration of data acquisition, recording, and transmission.

时网络传输和硬盘记录，可靠性受到限制，使用维护 不便.

在嫦娥五号(CE-5)以前的任务中，VLBI观测目标 仅为一个月球探测器的信号, 而CE-5任务中, VLBI观 测目标包含了多个目标探测器，尤其是在月球轨道交 会对接等关键弧段, VLBI采用同波束模式同时接收轨 道器和上升器的各种测控与数传信号. 最多的时候, 需 要采样记录 22 个点频, 且频谱形式多、动态范围大, 实 时性、可靠性、测量精度要求高.

为满足嫦娥五号动态双目标同波束VLBI测量及 未来深空探测VLBI测轨需求, 我们以前期终端研制技 术为基础，开展了 16 通道、多比特信号采集、记录与 传输一体化技术的研究，研制了深空一体化终端，如 图1(c)所示. 不仅减小了基带通道间的仪器时延, 提高 了相位稳定性及测量精度, 还具备了VLBI数据采集、 记录、传输一体能力, 提高了可靠性, 并且增加了多比 特量化功能.
本文介绍了VLBI多通道多比特信号采集记录与 传输一体化技术及其在嫦娥五号任务中的应用情况.

\section{2 多通道多比特VLBI信号采集记录与传输 一体化技术}

多通道多比特VLBI信号采集记录与传输一体化 技术可以针对深空探测VLBI测轨需求, 将原来多个设 备模块才能完成的功能集中在一套设备中予以实现. 由于利用了超大规模可编程门阵列(Field Programmable Gate Array, FPGA)芯片内的丰富资源, 将数字下变 频、格式封装以及网络传输功能集成在一片芯片中, 不仅提高了相位测量的一致性和稳定性，还最大程度 地提高了集成度, 提升可靠性.

\section{1 可变频率多项滤波器组下变频算法}

在传统的射电天文VLBI终端中, 多采用数字直接 
下变频(Digital Down Converter, DDC $)^{[16]}$ 或者基于多项 滤波器组(Polyphase Filter Bank, PFB) ${ }^{[17]}$ 的下变频两种 算法. 前者的优点是每个输出通道的频率可精确设置, 通道带宽可变，但算法结构复杂．而后者虽然结构简 单、效率高, 但频率不可调整.

在大地测量的VLBI观测中，其观测信号为宽带白 噪声，对频率没有特殊要求，只需要各站频率一致即 可，因此PFB算法在大地测量的VLBI终端中得到了广 泛应用. 而深空探测中, 观测信号来自探测器信标, 每 个探测器都不相同. 因此观测通道频率需根据不同探 测器的信标频点进行灵活设置.

多通道多比特VLBI信号采集记录与传输一体化 技术中, 使用了可变频率的多项滤波器组算法. 该算法 综合了PFB和DDC两种算法的特点，平衡了资源和算 法效率，实现了多种带宽下16通道的可调频率的数字 下变频，减小了通道间的时延差异. 同时还增加了针 对探测器信号的窄带多比特量化模式，可以进一步提 高信噪比和网络带宽利用率.

可变频率多项滤波器组下变频算法分为两级，第 一级采用PBF方法, 用矩形系数为 2 的滤波器, 对中 频信号进行信道划分，每个通道的中心频率为 $\omega_{k}=$ $\frac{2 \pi}{M} k+\frac{\pi}{2 M}$, 带宽为 $\frac{2 \pi}{M}$.

由于实信号的频谱具有对称性, 因此, 这种划分方 法得到的信道相互之间有交叠，能够覆盖整个输入频 带，不存在盲区，如图2(a)所示. 图中虚线部分信道 $\left(k=\frac{M}{2}, \cdots M-1\right)$ 为 $(\pi, 2 \pi)$ 范围内对应信道的镜像.

当采用临界抽取 $D=M$ 时, 有

$$
\begin{aligned}
y_{k}(m) & =\sum_{p=0}^{M-1}\left\{\left[x_{p}(m) \mathrm{e}^{-\mathrm{j} \frac{\pi}{2} m}\right] * g_{p}(m)\right\} \mathrm{e}^{\mathrm{j} \frac{\pi}{2 M} p} \mathrm{e}^{-\mathrm{j} \frac{2 \pi}{M} k p} \\
& =D F T\left(\left\{\left[x_{p}(m) \mathrm{e}^{-\mathrm{j} \frac{\pi}{2} m}\right] * g_{p}(m)\right\} \mathrm{e}^{\mathrm{j} \frac{\pi}{2 M} p}\right),
\end{aligned}
$$

其中, $x_{p}(m)=x(m M-p)$, 即原始信号的多相延时分量, $g_{p}(m)$ 为原型低通滤波器的多相分支滤波器, $y_{k}(m)$ 的 多相结构如图2(b)所示.

第二级采用DDC方法，从多相滤波器输出的多个 信道中, 选择包含目标频率的 $N$ 个信道, 分别进行下变 频和数字滤波. 滤波器采用级联的方式, 后一级滤波器 带宽为前一级的 $1 / 2$, 根据所需要的带宽, 选择从哪一 级滤波器输出基带信号, 如图2(c)所示.
在FPGA实现中，PFB用来降低数据率和时钟速 率，DDC用来调整频率和带宽，两者结合可实现频率 可调、带宽可变的下变频处理.

为保证精度, 处理时采用了 16 bit定点运算, 因此, 生成的基带信号原始字长为 16 bit. 在输出时，若使用 传统的2 bit量化模式, 则需要采用阈值比较 ${ }^{[18]}$ 的方法, 以达到 $00,01,10$ 和 11 四种状态之间的比例分别为 $18 \%$, $32 \% ， 32 \%$ 和 $18 \%$ 的要求 ${ }^{[19]}$ ，若使用多比特 ${ }^{[20]}$ 模式时,

(a)
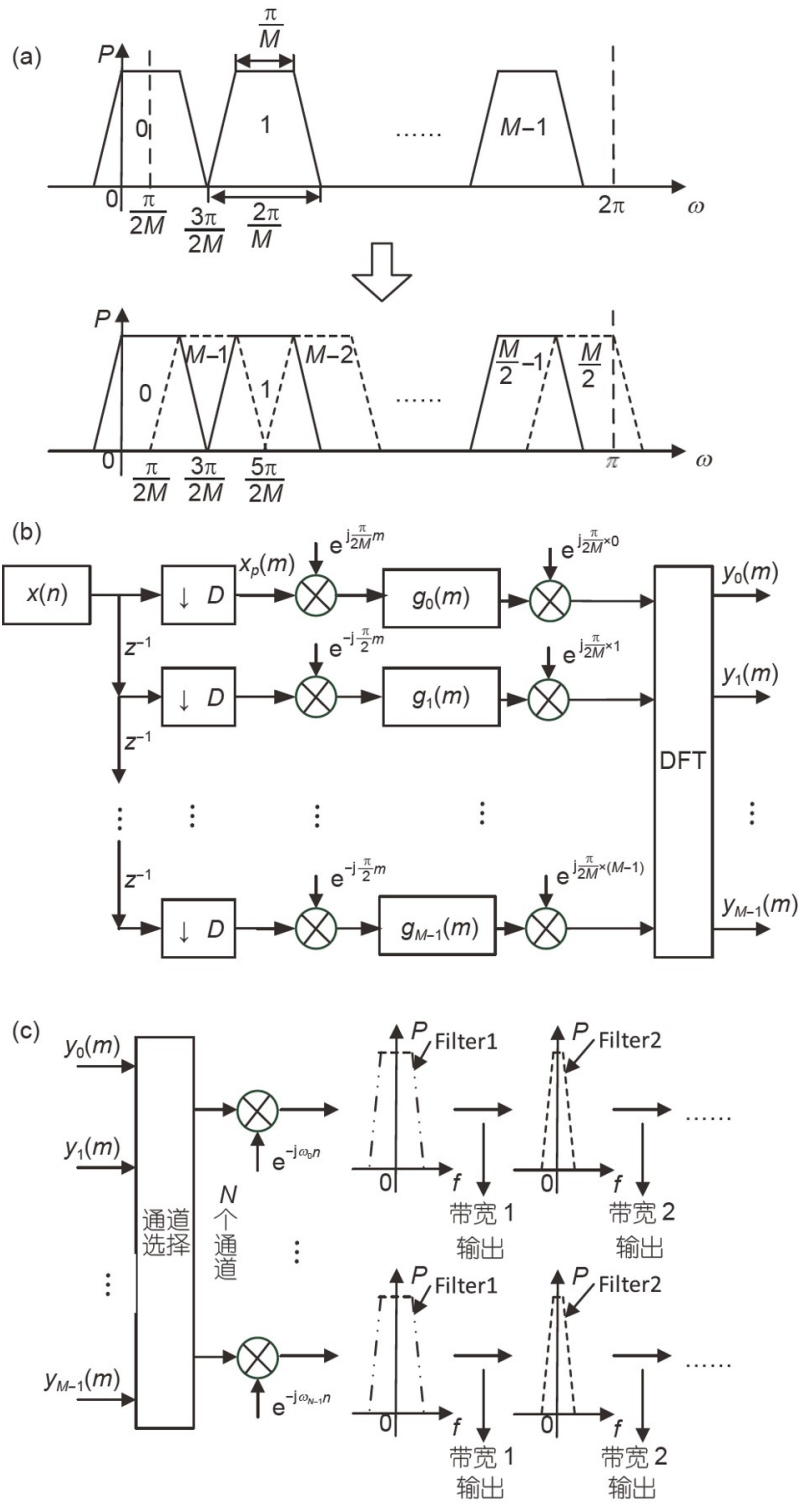

图 2 可变频率多项滤波器组算法框图. (a) 实信号信道划 分示意图; (b) 信道划分的多相结构; (c) 下变频

Figure 2 The diagram of tunable PFB. (a) Diagram of real signal channel division; (b) polyphase based channel division; (c) down conversion. 
则直接截取高位.

\section{2 数据格式封装和网络传输}

VLBI基带数据在记录之前, 要按照一定的格式, 插入同步字、时间码等信息，封装成数据帧，这项功 能过去是在Mark5B记录设备里完成. 而Mark5B记录 设备依赖进口，故障率高，而且本地数据记录和网络 传输两者不能同时进行，在实时任务中，一旦网络发 生故障, 必然会发生观测数据丢失.

与之相比，多通道多比特VLBI信号采集记录与传 输一体化技术将数据格式封装功能、网络传输功能 (图3(a))集成在采集设备中, 经过封装的数据可以直接 接入网络. 采用商用服务器替代Mark5B数据记录, 如 图3(b)所示，能够一边记录，一边传输，可提高整个 VLBI观测系统的可靠性和稳定性.

为支持多比特量化模式, 数据封装还对VSI (VLBI Standard Interface, https://ivscc.gsfc.nasa.gov/publications/ar2002/spcl-vsi-s_rev1.pdf)标准进行了扩展定义, 不仅支持最大 16 通道 2 bit量化模式, 还支持最大 8 通道 4 bit量化、4通道8 bit量化和 2 通道16 bit量化等模式.

\section{3 深空探测VLBI一体化终端}

CDAS2-D就是根据上述算法, 采用ADC+FPGA构 架研制的深空探测一体化终端，其系统结构框图分别 如图4所示.

图中, $10 \mathrm{MHz}$ 标准频率经频率综合器, 为ADC提 供采样时钟，中频信号经过信号调理电路，为 $\mathrm{ADC}$ 提 供最佳量化值 ${ }^{[21]}$ ，量化后的数字化中频信号，进入 FPGA进行处理, 生成VSI格式的网络数据帧输出,
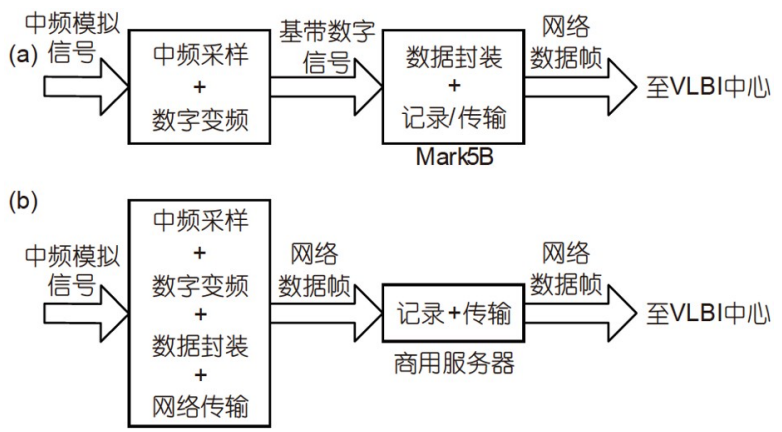

图 3 数据封装和网络传输功能集成在专用记录设备(a)和 VLBI一体化终端(b)中

Figure 3 Data encapsulation and network transmission functions in dedicated recorder (a) and integrated VLBI backend (b).
1PPS提供同步脉冲. 输出的数据由记录服务器进行记 录, 记录服务器可以事后或实时地将数据再次分发.

CDAS2-D支持 S/X两个频段的中频同时采样，每 个频段 $512 \mathrm{MHz}$ 带宽, 最大 16 个基带子通道输出; 带宽 32/16/8/4/2/1 MHz可选, 频率步进 $10 \mathrm{kHz}$. 支持 $1 / 2 / 4 / 8 /$ 16 bit量化, 采用VSI数据格式, 万兆以太网输出, 最大 码率 2048 Mbps. 带宽、量化比特、最大通道数和数据

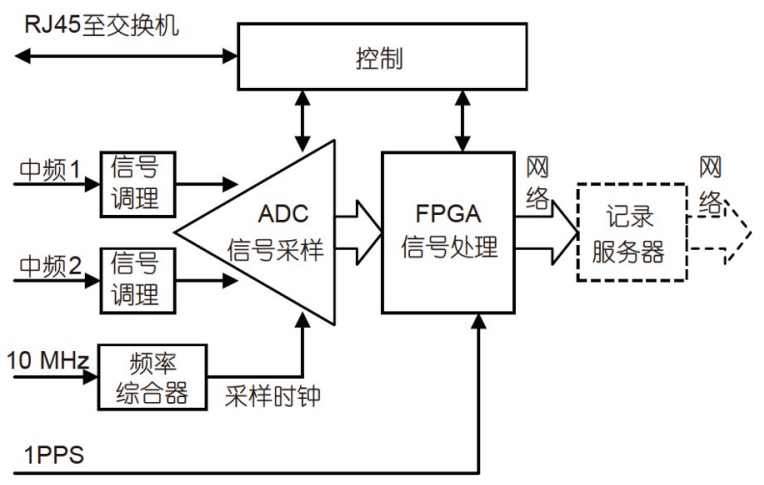

图 4 深空探测VLBI一体化终端结构框图

Figure 4 Diagram of CDAS2-D.

表 2 深空一体化终端工作模式

Table 2 Supported mode for CDAS2-D

\begin{tabular}{|c|c|c|c|}
\hline 带宽 (MHz) & 量化比特 (bit) & 最大子通道数 & 数据速率 (Mbps) \\
\hline \multirow{2}{*}{32} & 1 & 16 & 1024 \\
\hline & 2 & 16 & 2048 \\
\hline \multirow{2}{*}{16} & 1 & 16 & 512 \\
\hline & 2 & 16 & 1024 \\
\hline \multirow{2}{*}{8} & 1 & 16 & 256 \\
\hline & 2 & 16 & 512 \\
\hline \multirow{2}{*}{4} & 1 & 16 & 128 \\
\hline & 2 & 16 & 256 \\
\hline \multirow{5}{*}{2} & 1 & 16 & 64 \\
\hline & 2 & 16 & 128 \\
\hline & 4 & 8 & 128 \\
\hline & 8 & 4 & 128 \\
\hline & 16 & 2 & 128 \\
\hline \multirow{5}{*}{1} & 1 & 16 & 32 \\
\hline & 2 & 16 & 64 \\
\hline & 4 & 8 & 64 \\
\hline & 8 & 4 & 64 \\
\hline & 16 & 2 & 64 \\
\hline \multirow{5}{*}{0.5} & 1 & 16 & 16 \\
\hline & 2 & 16 & 32 \\
\hline & 4 & 8 & 32 \\
\hline & 8 & 4 & 32 \\
\hline & 16 & 2 & 32 \\
\hline
\end{tabular}


速率之间的关系如表2所示. 由于采用了以太网接口， 终端输出数据可通过交换机，或者以点对点的方式， 接到商用服务器进行数据记录.

在过去的CDAS终端中, 由于硬件结构的原因, 16 个基带通道分别由 16 片FPGA芯片独立处理，芯片与 芯片之间的数据传输、数据同步都存在不同程度的时 延, 导致通道之间时延差异较大, 达到百纳秒级. 深空 一体化终端CDAS2-D针对这一问题，从算法设计和硬 件结构两方面做了改进. 一方面改进算法, 在性能和资 源之间进行平衡，提高资源利用率；另一方面，使用容 量更大、资源更多的FPGA芯片.

经过改进, CDAS2-D能将原来CDAS中由多芯片 完成的工作集成到一片芯片中. 16 个基带通道的处理 可以在一片FPGA芯片中完成，集成度的提高使得基 带子通道之间时延的差异有了数量级的减小.

图5和6分别是嫦娥三号任务(使用CDAS终端)和嫦 娥五号任务(使用一体化CDAS2-D终端)中通过射电源观 测获得的终端通道互相关特性. 嫦娥三号和嫦娥五号任 务中, 探测器DOR信号均在X频段, 终端输出的基带带宽 均为 $2 \mathrm{MHz}$ 通道. 图中蓝色表示幅频特性, 红色表示相频
特性也称为VLBI相关条纹，条纹的斜率代表通道时延. 从幅频特性来看，两者略有差异. CDAS2-D终端的过渡 带略大, 这是滤波器设计中, 性能和资源平衡的结果, 用 牺牲滤波器级数的代价节省资源, 来达到容纳更多的通 道, 提升集成度的目的. 从相频特性本身来看, 两者差异 不明显, 都保持了线性相位, 而从通道时延来看, CDAS2$\mathrm{D}$ 终端通道时延的变化明显小于CDAS终端.

图7给出了两者通道时延的比较, 蓝色代表CDAS 终端, 红色代表CDAS2-D终端, 横轴表示不同通道. 通 过均值和方差的计算可以看出, CDAS终端通道间时 延的均方差为 $120.4 \mathrm{~ns}$, 而CDAS2-D终端通道间时延 均方差仅为 $1.21 \mathrm{~ns}$, 比CDAS终端减小了 2 个数量级.

CDAS2-D还增加了多比特量化输出功能. 由于量 化噪声的原因, 低比特量化方式对测控载波、DOR侧 音等点频信号，会产生多个假频率，不仅会造成DOR 信号失真, 而且也会降低真实点频信号的信噪比. 而 8 bit或16 bit量化模式下, 能够很好地还原原始点频信 号, 减少信噪比损失, 还能针对性地对点频附近信号进 行窄带采样, 大大提高采样效率. 未来的深空探测中, 可以采用以宽带低比特量化方式采集射电源信号, 以
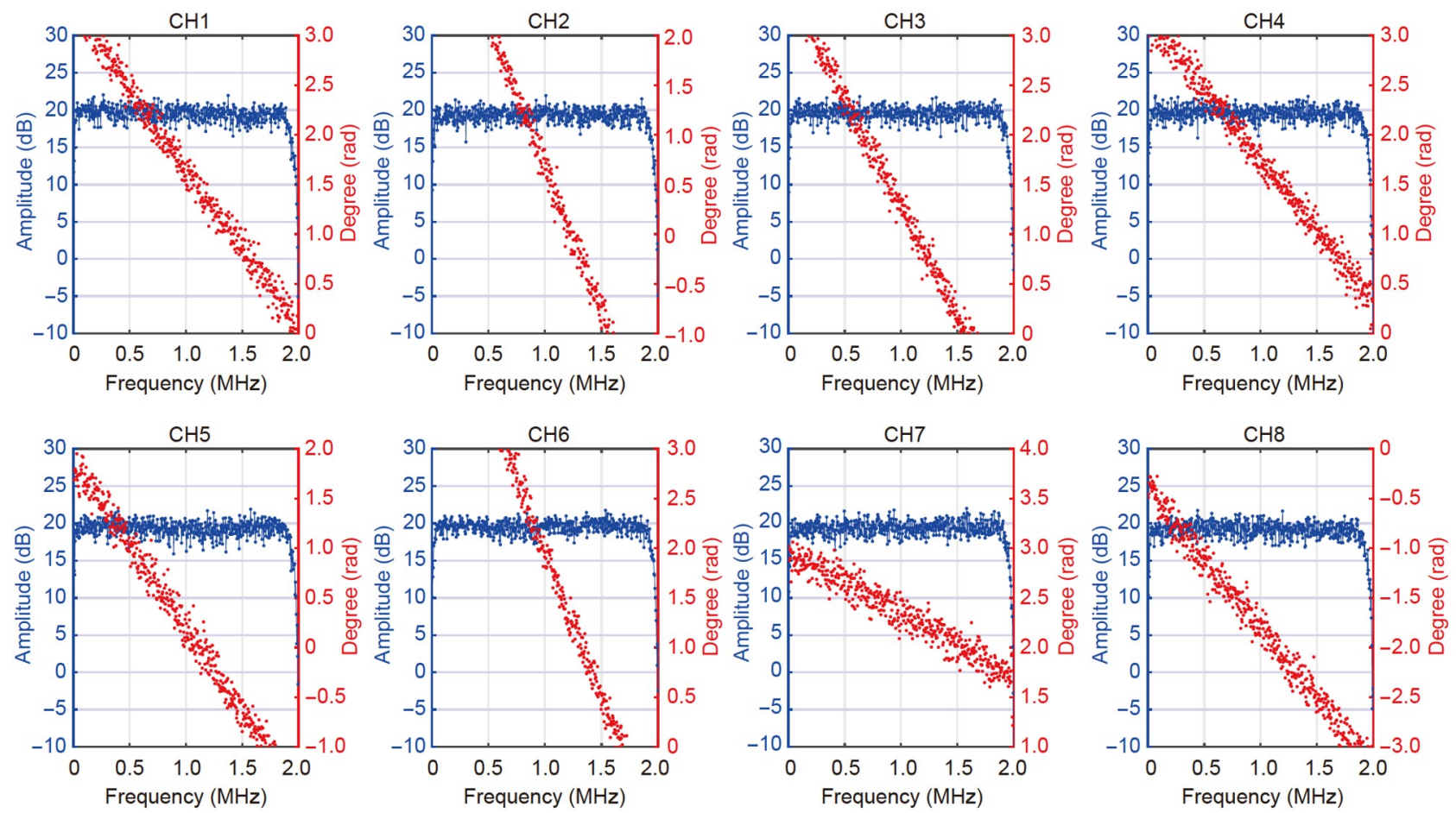

图 5 (网络版彩图)原有CDAS带通特性

Figure 5 (Color online) Band-pass characteristics of CDAS. 

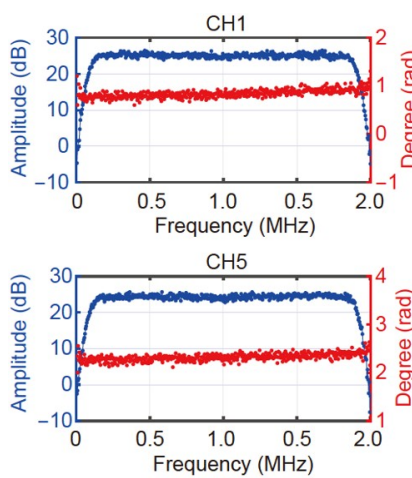

$\mathrm{CH} 9$

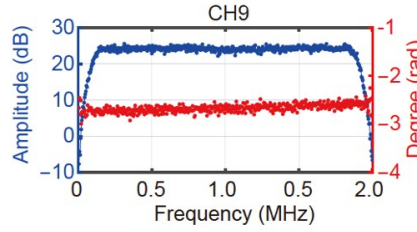

$\mathrm{CH} 13$
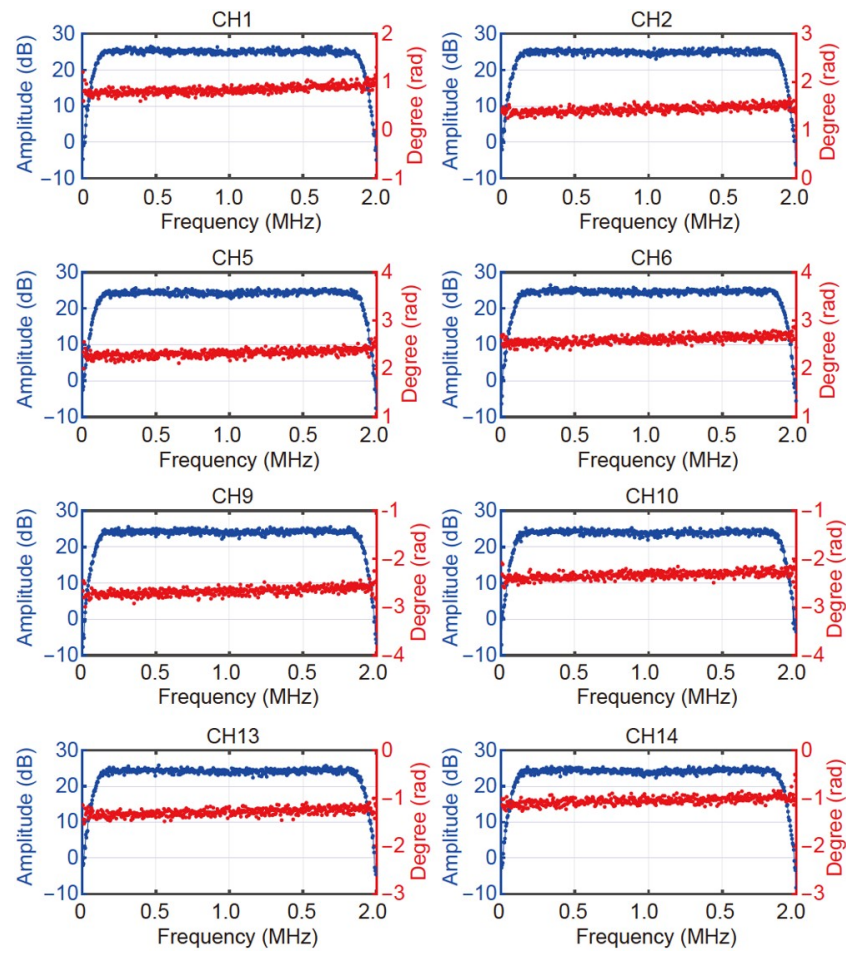

$\mathrm{CH} 10$
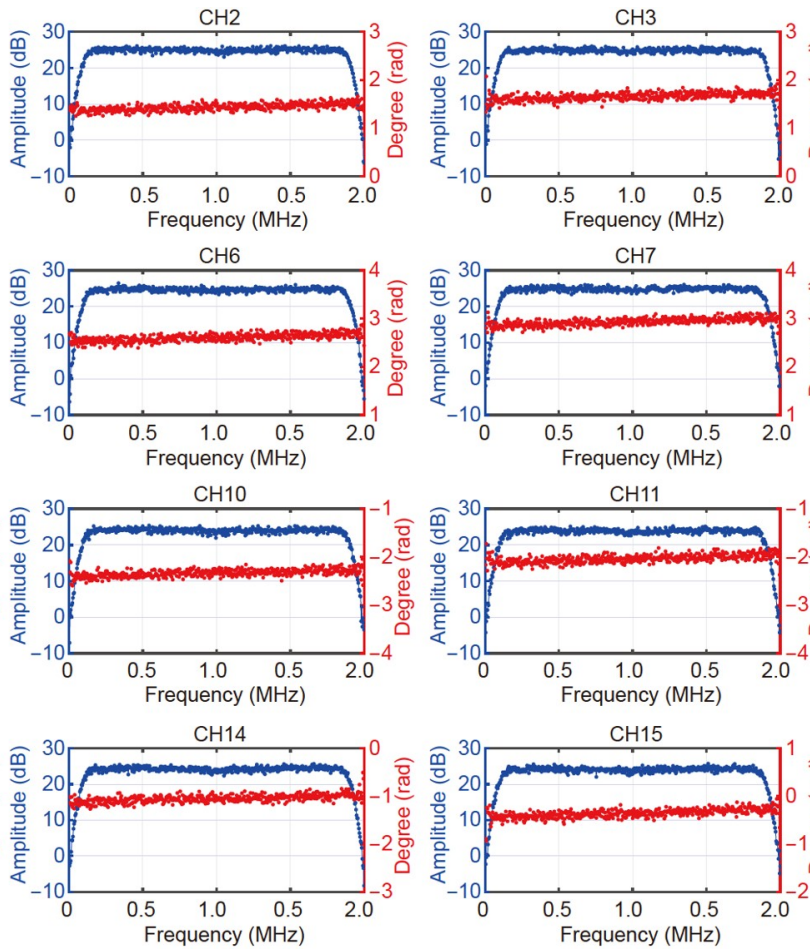

$\mathrm{CH} 7$

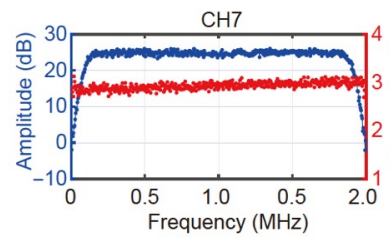

$\mathrm{CH} 11$

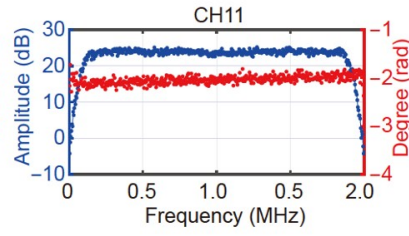

$\mathrm{CH} 15$
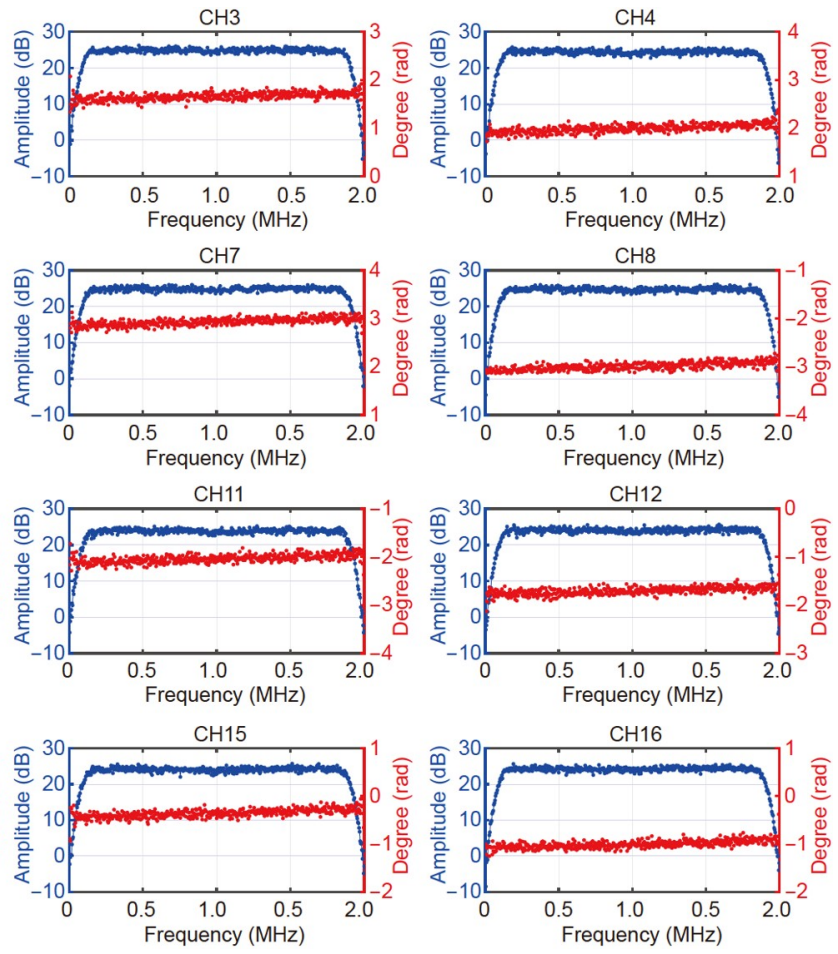

图 6 (网络版彩图)新型CDAS2-D带通特性

Figure 6 (Color online) Band-pass characteristics of CDAS2-D.

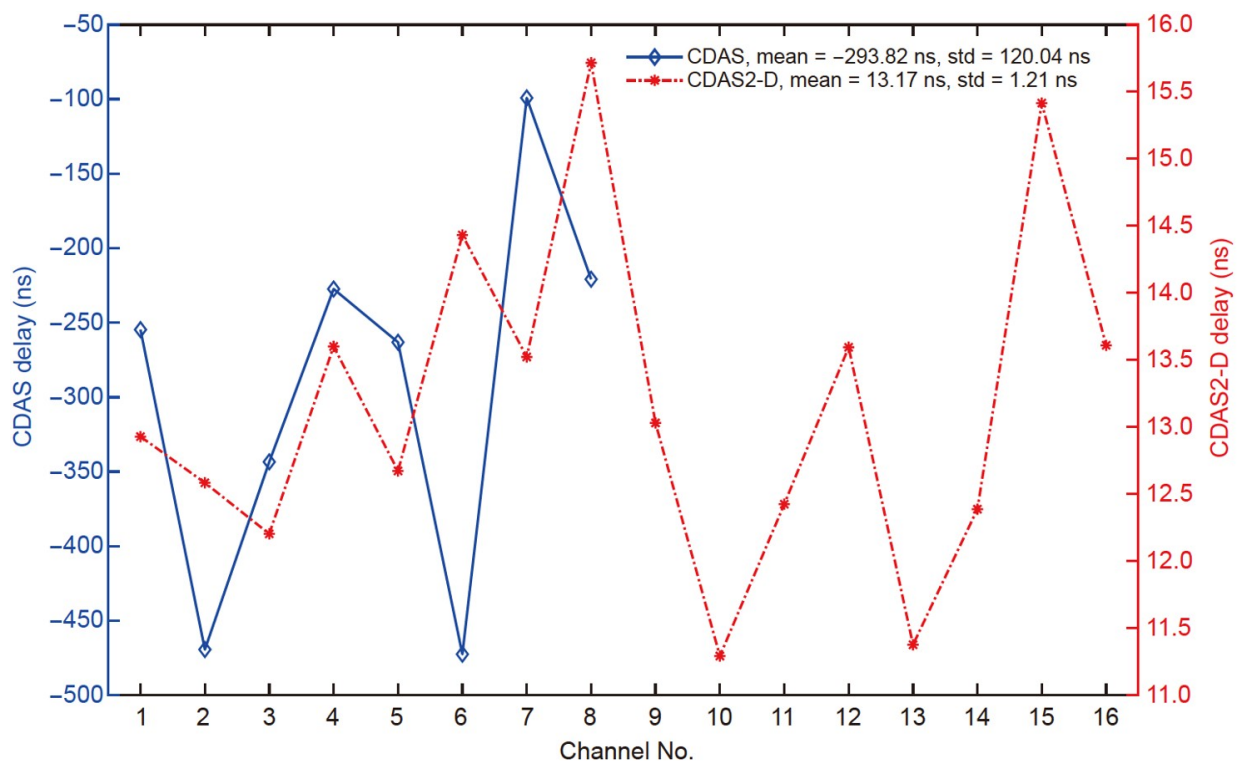

图 7 (网络版彩图)多通道时延比较

Figure 7 (Color online) Comparison of multi-channel delay.

窄带多比特方式采集探测器信号. 图8给出了DOR信 号经2 bit和 8 bit量化后的差异. 图8(a)是2 bit量化的结
果，图中红色部分是由于 2 bit量化的能量泄露引入的 假频，图8(b)是采用了 8 bit量化的结果，可以看出 
8 bit量化的结果明显优于 2 bit量化结果.

\section{3 嫦娥五号任务中的应用}

嫦娥五号共有 4 组DOR信标和 2 个数传信号. 每组 DOR信标由主载波和与主载波频率相差 $\pm 3.8 \mathrm{MHz}$ 和 $\pm 19.2 \mathrm{MHz}$ 的两组侧音构成，即每组DOR信标有 5 个频 点, 4 组共 20 个频点, 再加上 2 个数传信号, 总共是 22 个 频点, 这22个频点的相对位置如图9所示. 其中FnPC表 示第 $n$ 组DOR信号的主载波频率, FnL1, FnR1, FnL2和 FnR2依次表示第 $n$ 组DOR信号中，与主载波频率相差 $-3.8,+3.8,-19.2$ 和 $+19.2 \mathrm{MHz}$ 的四个侧音信号频率, F5 和F6是数传信号.

在嫦娥五号任务中，探测器动作多，信标切换频 繁. 由于轨道及探测器姿态等原因，信标状态不可能 预先精确知晓，为确保始终有可用的信标，必须实 时、完整地记录所有可用于VLBI观测的信标.

在实际观测中, 利用 $2 \mathrm{MHz}$ 带宽的观测模式, 通过

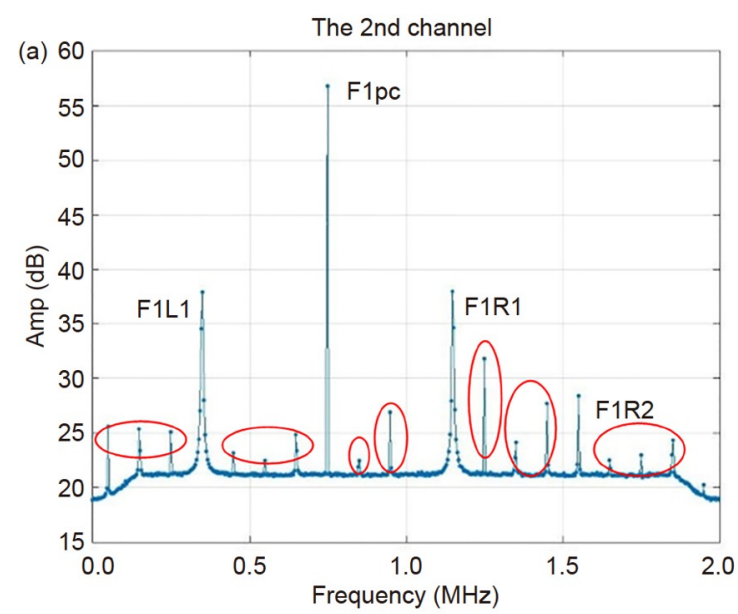

巧妙的频点设置, 可用终端的 16 个基带通道覆盖所有 22 个频点(图9中的黑框所示). 有些相距很近的频点, 被设置在一个基带通道里. 比如第 6,10 通道，各包含 了 3 个频点, 第 $4,8,12$ 通道, 各包含了 2 个频点, 第 16 通 道与第 3 通道联合, 覆盖数传信号.

图10和 11 是天马-南山基线VLBI相关处理后的 16 通道探测器信号的频谱和相关条纹, 其中第 $7,11,5,9$ 通道依次对应F1-F4的4组DOR的主载波. 从图10中 可以看到，该时刻4组DOR均可见，且测速测距上行频 率锁定F3频点. 因此，在与F3有关的DOR信标两侧约 $500 \mathrm{kHz}$ 处均出现了测距信号, 见图10中第1, 4, 5, 6, 10 通道. 此时探测器无数传任务, 因此第 3,13 和 16 通道未 见数传信号.

由于采用了 16 通道全信标覆盖的观测模式, CDAS2-D终端同时记录了全部探测器信号, 当探测器 工作状态发生变化, 星上信标切换时, 测站不需要切换 观测模式, 从而保证了VLBI观测的连续性、实时性和 可靠性. VLBI中心也能够实时地了解全部信标的状

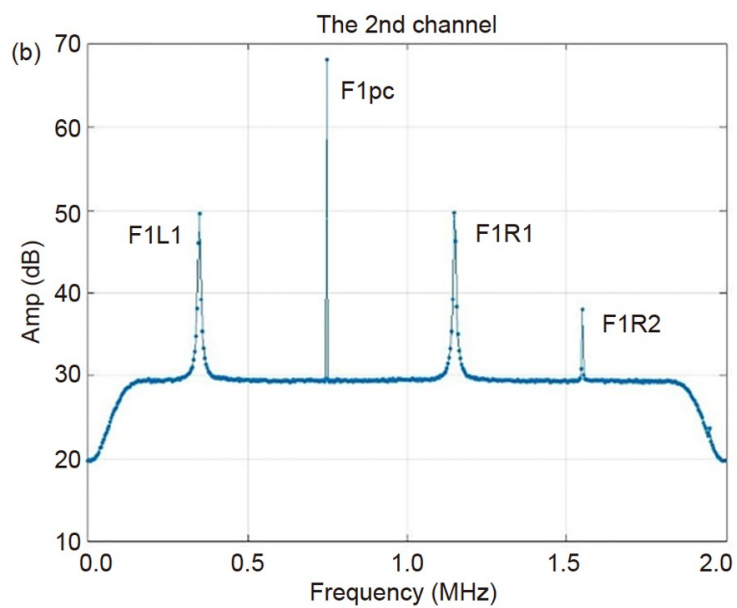

图 8 (网络版彩图)不同量化模式之间的比较. (a) 2 bit采样(红圈部分为假频); (b) 8 bit采样

Figure 8 (Color online) Comparison between different quantization models. (a) The 2-bit quantization (alias frequency marked by circle); (b) the 8bit quantization.

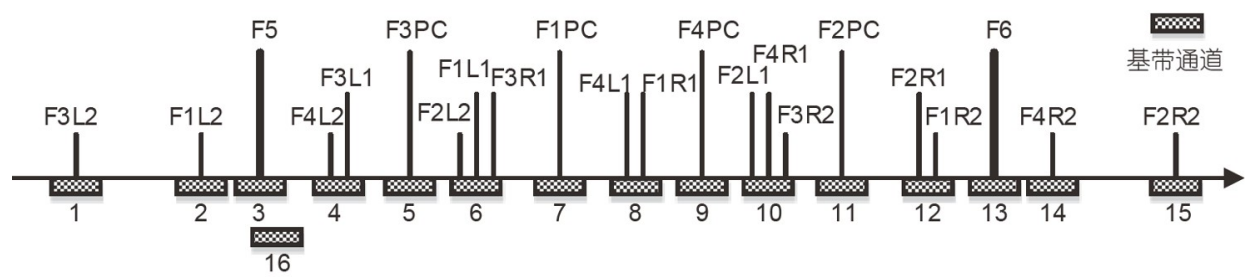

图 9 嫦娥五号探测器DOR信标及通道分布

Figure 9 DOR beacons and channel distribution of Chang'e-5 prober. 

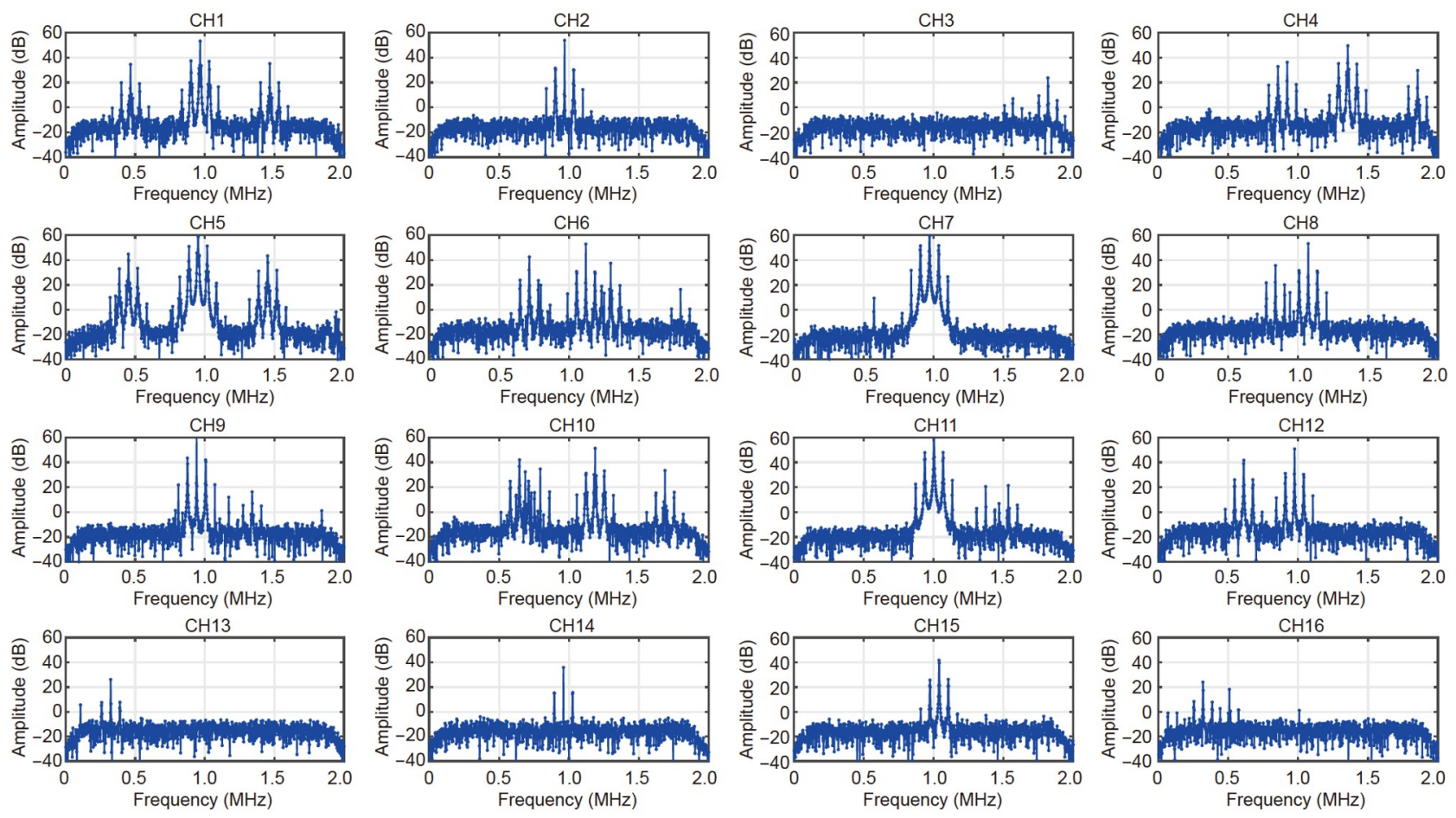

图 10 (网络版彩图)天马-南山基线嫦娥五号信标VLBI相关处理频谱

Figure 10 (Color online) VLBI related processing spectrum of Chang'e-5 beacon from Tianma-Nanshan baseline.
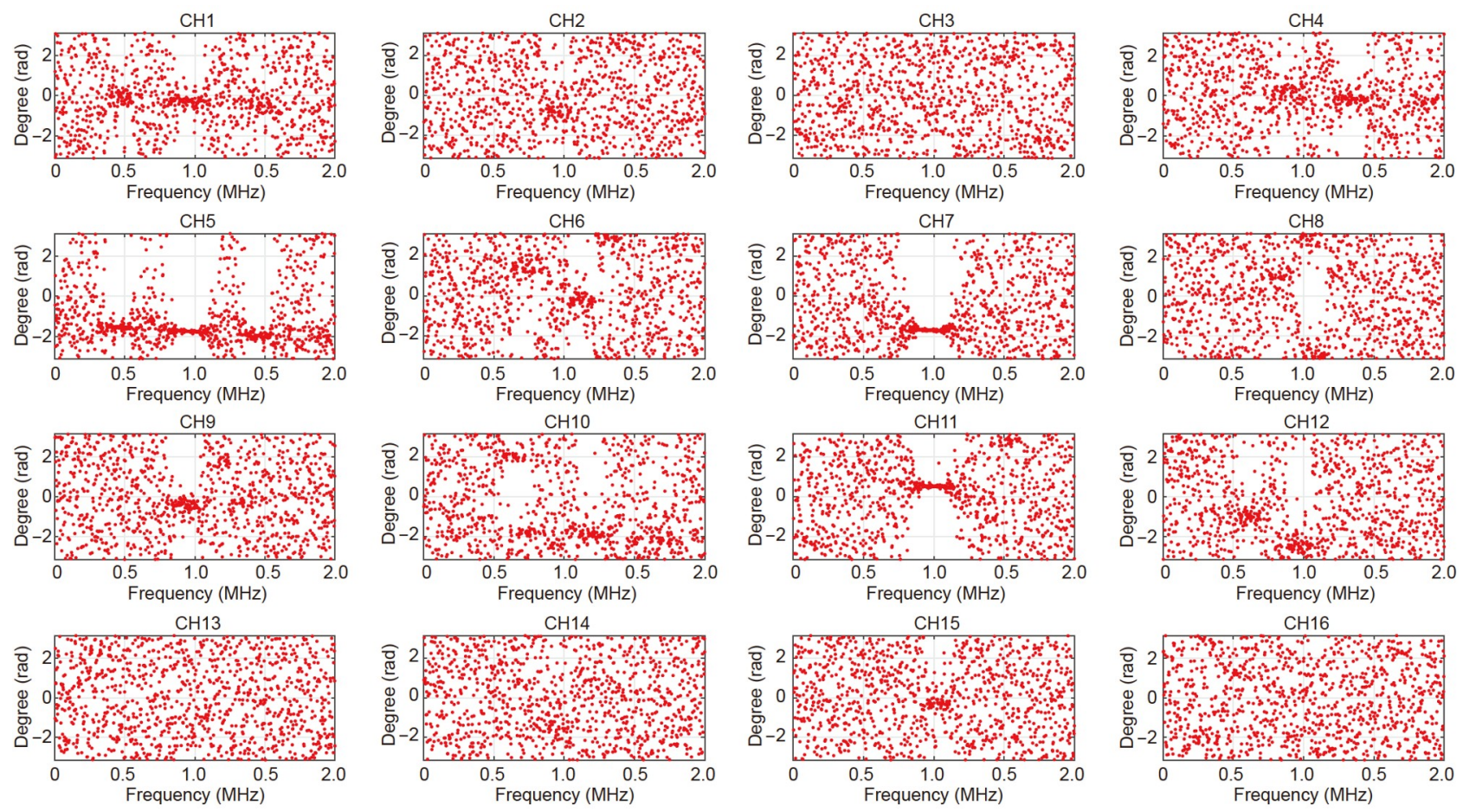

图 11 (网络版彩图)天马-南山基线嫦娥五号信标VLBI相关处理移条纹

Figure 11 (Color online) VLBI related processing fringes of Chang'e-5 beacon from Tianma-Nanshan baseline. 
态, 融合多个信标的最优测量结果, 用于测定轨, 确保 了双目标交会对接等高难度测轨任务的完成.

\section{4 结论}

针对CE-5多探测器VLBI测轨需求, 我们开展了多
通道多比特信号采集、处理与传输一体化技术研究, 并研制了新型深空专用CDAS2-D终端. 终端结构紧凑, 性能与可靠性有显著提高, 支持数据实时记录和数据 传输，还具备多比特量化功能. 未来还可应用于其他 月球与行星探测VLBI测轨工作.

\section{参考文献}

1 Thompson A R, Moran J M, Swenson G W, et al. Interferometry and Synthesis in Radio Astronomy. 2nd ed. Hoboken: John Wiley \& Sons, Inc., 2001. 304

2 James U, Miller G. Radio astronomy's resolution machine: The very long baseline array. Sky Telescope, 1999, 98: 36-41

3 Yin N, Zhang X Z. The development of VLBI standard interface (in Chinese). Prog Astron, 2007, 25: 74-83 [殷妮, 张秀忠. VLBI标准接口的发 展. 天文学进展, 2007, 25: 74-83]

4 Neill A, Bark M, Beaudoin C, et al. RDBE Development and Progress. Technical Report. 6th International VLBI Service for Geodesy and Astronomy, 2010

5 Tuccari G. DBBC3 - A Full Digital Implementation of the VLBI2010 Backend. Technical Report. 7th General Meeting (GM2012) of the International VLBI Service for Geodesy and Astrometry, 2012

6 Takefuji K, Takeuchi H, Tsutsumi M, et al. Next-generation A/D Sampler ADS3000+ for VLBI2010. Technical Report. 6th International VLBI Service for Geodesy and Astronomy, 2010

7 Zhu R J, Zhang X Z, Wei W R, et al. The progress of modern Chinese data acquisition system (in Chinese). Prog Astron, 2011, 29: 207-217 [ 人杰, 张秀忠, 韦文仁, 等. 我国新一代VLBI数字基带转换器研制进展. 天文学进展, 2011, 29: 207-217]

8 Wei S J, Hou X M, Ma H, et al. Research on the development status of deep space TT\& C VLBI digital baseband converter (in Chinese). J Telemetry, Track Command, 2014, 35: 1-9 [魏绍杰, 侯孝民, 马宏, 等. 深空测控VLBI数字基带转换器发展现状研究. 遥测遥控, 2014, 35: 19]

9 Akiyama K, et al. (The Event Horizon Telescope Collaboration). First M87 Event Horizon Telescope results. II. Array and instrumentation. Astrophys J Lett, 2019, 875: 8

10 Wu W R, Wang G L, Jie D G, et al. High-accuracy VLBI technique using $\Delta$ DOR signals (in Chinese). Sci Sin-Inf, 2013, 43: 185-196 [吴伟仁, 王 广利, 节德刚, 等. 基于 $\triangle \mathrm{DOR}$ 信号的高精度VLBI技术. 中国科学: 信息科学, 2013, 43: 185-196]

11 Thornton C L, Border J S. Radiometric Tracking Techniques for Deep-Space Navigation. Hoboken: John Wiley \& Sons, Inc., 2003. 65-66

12 Madde R, Morley T, Lanucara M, et al. A common receiver architecture for ESA radio science and delta-DOR support. Proc IEEE, 2007, 95: 2215-2223

13 Whitney A. The Mark 5 VLBI Data System. Technical Report. The 18th European VLBI for Geodesy and Astrometry Work Meeting, 2007

14 Whitney A. The Mark 5B VLBI Data System. Technical Report. The 7th Symposium of the European VLBI Network on New Developments in VLBI Science and Technology, 2004

15 Zhu R J, Wu Y J. Adjustment for the delay alignment among channels of Chinese VLBI data acquisition system (in Chinese). Prog Astron, 2014, 32: 118-127 [朱人杰, 吴亚军. VLBI数字基带转换器通道时延一致性的调整. 天文学进展, 2014, 32: 118-127]

16 Xiang Y, Zhu R J, Tuccari G, et al. Wideband digital SSB down converter (in Chinese). Acta Electron Sin, 2006, 34: 1978-1980 [项英, 朱人杰, Tuccari G, 等. 宽带数字单边带下变频器. 电子学报, 2006, 34: 1978-1980]

17 Chen L, Zhang X Z. The study of DBBC based on poly-phase filter banks and FFT in VLBI (in Chinese). Prog Astron, 2008, 26: 87-94 [陈岗, 张 秀忠. 用于VLBI数字基带转换的多相滤波技术研究. 天文学进展, 2008, 26: 87-94]

18 Zhang B J, Wu Y J, Yu W, et al. A method of calculating the quantization threshold for a VLBI DBBC and its FPGA implementation (in Chinese). Astronom Res Technol, 2013, 10: 219-226 [张碧娟, 吴亚军, 于威, 等. 一种VLBI数字基带转换器的阈值计算方法及其FPGA实现. 天文研究与技术, 2013, 10: 219-226]

19 Kokkeler A B J, Fridman P, van Ardenne A. Degradation due to quantization noise in radioastronomy phased arrays. Exp Astron, 2001, 11: 3356 


\title{
Integration of very long baseline interferometry data acquisi- tion, recording, and transmission with multichannel and multibit for CE-5 mission
}

\author{
ZHU RenJie ${ }^{1,2,3,4}$, WU YaJun ${ }^{1,2}$, ZHENG WeiMin ${ }^{1,2,3,4^{*}}$, LI JiYun ${ }^{1}$, GUO ShaoGuang ${ }^{1,2}$, \\ ZHANG XiuZhong ${ }^{1} \&$ WANG GuangLi ${ }^{1,4}$ \\ ${ }^{1}$ Shanghai Astronomical Observatory, Chinese Academy of Sciences, Shanghai 200030, China; \\ ${ }^{2}$ Key Laboratory of Radio Astronomy, Shanghai Astronomical Observatory, Chinese Academy of Sciences, Nanjing 210023, China; \\ ${ }^{3}$ National Basic Public Science Data Center, Beijing 100190, China; \\ ${ }^{4}$ Shanghai Key Laboratory of Space Navigation and Positioning Techniques, Shanghai Astronomical Observatory, \\ Chinese Academy of Sciences, Shanghai 200030, China
}

Real-time signal acquisition, recording, and transmission are the important functions of very long baseline interferometry (VLBI). In the Chang'e-5 (CE-5) mission, a VLBI backend is required to connect the telemetry, track and command (TT\&C) beacons, data signals from multiple probes, such as the orbiter, ascender, and lander, and signals from the extragalactic radio source in time. Due to the multiple frequency points, large dynamic range, and complex spectrum form of the probe signals, existing VLBI astronomical backends do not meet the requirements of lunar exploration and deep space exploration. According to the characteristics of the beacon of CE-5 probes, we researched the integration technology of multichannel and multibit signal acquisition, recording, and transmission. This paper proposes a frequency tunable polyphase-filter bank algorithm, which can flexibly set frequency and obtain flat amplitude and linear phase characteristics in a wide band. The novel integrated VLBI backend developed by this technology contains 16 channels, which can sample and record all the TT\&C and data signals from CE-5 probes in real time and transmit the VLBI data to the VLBI center through the network. It ensures successful VLBI measurement in the complex electromagnetic environment of CE-5 multi beacons. Compared with the previous astronomical backends, the real-time performance, measurement accuracy, and reliability of the novel integrated VLBI backend are greatly improved, which support the successful completion of China's first lunar sampling return mission of CE-5. The novel integrated VLBI backend also has the function of multibit quantization to meet the needs of wide and narrow observation of future radio sources and probes.

VLBI, CE-5, frequency tuneable polyphase filter bank, delay, integrated backend

PACS: $95.55 . \mathrm{Br}, 95.55 . \mathrm{Jz}, 95.55 . \mathrm{Pe}, 95.75 . \mathrm{Wx}$

doi: $10.1360 /$ SSPMA-2021-0076 